\title{
EL SUJETO INFORMACIONAL EN EL CONTEXTO CONTEMPORÁNEO. UN ANÁLISIS DESDE LA EPISTEMOLOGÍA DE LA IDENTIDAD COMUNITARIA- INFORMACIONAL
}

\author{
Miguel Angel Rendón-Rojas ${ }^{\mathrm{i}}$ \\ Alejandro García-Cervantes ${ }^{\text {ii }}$
}

\begin{abstract}
Resumen: Se propone la Epistemología de la Identidad Comunitaria-Informacional (EIC-I) como caja de herramientas para el análisis teórico-metodológico de la realidad informativa, por medio de sus categorías construidas ex profeso: paradigma contextual, sujeto informacional, entidades de información. Se distinguen los conceptos de usuario de la información y sujeto informacional. Éste último se autoconstruye en un enclave social concreto dentro de su comunidad e interrelaciones con los "otros", como interpelación al mundo desigual, consumista y alienante, desde donde surgen sus necesidades de información concretas para responder, cuestionar y actuar en ese mundo. Se enfatiza la necesidad de un acercamiento interdisciplinar en el estudio del mundo informativo documental entre la teoría social y la bibliotecología para conocer ese sujeto informacional concreto, muchas veces marginado y excluido.
\end{abstract}

Palabras-clave: Epistemología de la bibliotecología. Epistemología de la Identidad Comunitaria-Informacional. Usuario. Sujeto informacional. Entidades de información.

THE INFORMATIONAL SUBJECT IN THE CONTEMPORARY CONTEXT. AN ANALYSIS FROM THE EPISTEMOLOGY OF INFORMATIONAL COMMUNITY IDENTITY

\begin{abstract}
The Epistemology of Informational Community Identity (ECI-I) is proposed as a toolbox for the analysis of informational reality within categories as contextual paradigm, informational subject and informational entity, built ex profeso for this theoretical-methodological analysis. The concepts of information user's and informational subject are distinguished, the latest, to seek an answer from a concrete social enclave within a particular community and its interrelationships with others, to under go a process of self construction, from which specific information needs arise. And the user needs to seek concrete answer after formal questioning many facts occurring in a consumerist, unequal and alienating world. So the emphasis is put on the need for an interdisciplinary approach between social theory and library science in the study of the documentary information world of particular informational subjects, which is often marginalized and excluded.
\end{abstract}

Keywords: Epistemology of Library Science. Epistemology of Informational Community Identity. User. Informational subject. Informational entity.

\footnotetext{
${ }^{\mathrm{i}}$ Universidad Nacional Autónoma de México. marr@unam.mx.

${ }^{i i}$ Universidad Nacional Autónoma de México. ecosdnosfia@hotmail.com.

Recebido em: 16/11/2011; aceito para publicação em: 24/02/2012.
} 


\section{INTRODUCCIÓN}

El que hacer bibliotecológico tiene una historia que se remonta a la antigüedad y durante siglos su tarea ha abarcado a un conjunto de conocimientos técnicos y teóricos para la organización y administración de las unidades de información. Sin embargo no fue sino hasta finales del siglo XIX que las ciencias de la información comenzaron a desarrollar sus fundamentos epistémicos (BUSHA, HARTER, 1990). No obstante el trabajo desarrollado, aún es poco lo que se ha investigado sobre los fenómenos informativos en la constitución de la identidad de los individuos, los enclaves concretos y las relaciones sociales. Por lo anterior, es necesario, a partir de una teoría social, llenar esa laguna y estudiar esas relaciones sociales, el proceso cultural y los patrones de significado de la comunidad, incluidas las comunidades en situación de vulnerabilidad; para comprender la influencia del cambio social en las necesidades de información, reales y concretas de las sociedades contemporáneas.

\section{EPISTEMOLOGÍA DE LA IDENTIDAD COMUNITARIA: EXPLORACIÓN EN LOS CAMPOS EPISTÉMICOS DE LA PRAXIS PROFESIONAL E INVESTIGACIÓN BIBLIOTECOLÓGICA}

Para identificar esas tareas del cambio social en las necesidades de información utilizamos la Epistemología de la Identidad Comunitaria-Informacional (EIC-I) como caja de herramientas en el análisis teórico-metodológico concreto de esa realidad informativa compleja $^{3}$. La EIC-I es un corpus analítico que construye el saber, de ahí que es una epistemología, sobre la identidad de los sujetos en una comunidad concreta, por eso es de identidad comunitaria, y en su relación con el mundo informativo, por lo que adquiere el calificativo de informacional (GARCÍA CERVANTES, 2010, p. 139). La EIC-I tiene sus raíces en la epistemología social de Shera y Egan, además de un anclaje interdisciplinario sustancialmente filosófico, sociológico y antropológico. De manera muy general podemos decir que el objetivo de la epistemología social es identificar las fuerzas e influencias sociales que institucionalizan las creencias en comunidades, y determinan o condicionan las formas de producción, organización, circulación y uso del conocimiento (MORENO JIMÉNEZ, 2008).

\footnotetext{
${ }^{3}$ La noción $<<$ caja de herramientas $>>$ alude "al sentido de una analítica discursiva que involucra [...] procedimientos de investigación que retoma de diversas disciplinas [...] pone especial atención en la compatibilidad y/o compatibilización epistémica y ontológica de las herramientas intelectuales que articula, en busca de la mayor consistencia posible.". (BUENFIL BURGOS, 2008, p. 30)
} 
El uso de la EIC-I nos permite identificar, a partir del contexto, los momentos informacionales que intervienen en la emergencia de los que hemos denominado sujetos informacionales en situación de vulnerabilidad y marginación con respecto a la demanda concreta de la información en los enclaves socioculturales.

Si consideramos la praxis profesional como una intensión sociopolítica, entonces debemos analizar y comprender el entorno inmediato desde ella misma, a la vez de comprender el ambiente global en la que se encuentra inmersa. Lo anterior posibilita develar los sentidos ocultos de la modernidad -crisis de sentido- (BERGER, LUCKMANN, 1997; TOURAINE, 2000) y, de la posmodernidad, (LYOTARD, 1990, 1987; VATTIMO et al., 1990) la cual, aunque con vestiduras de novedad, continúa siendo neoconservadora e individualista dentro del esquema neoliberal que proclama la necesidad de producir y consumir conocimientos de moda o con caducidad. Así pues, se transita a otra episteme social construida desde la relación con el "otro", en una dialéctica social que permite difuminar el sentido consumista del binomio información-conocimiento en nuestra era.

La EIC-I constituye un andamiaje teórico para estudiar el sentido que se configura en las prácticas sociales de la triada dialéctica bibliotecológica: profesional de la informacióncomunidad-entidad de información. La EIC-I nos ayuda a construir un modelo contextual heurístico, una analítica social para acceder a un análisis ontológico, epistemológico y metodológico de las acciones socioinformativas, en el marco de la constitución de sujetos en el uso de la información en contextos vulnerables.

La EIC-I no busca realizar un análisis desde una perspectiva del orden institucional, sino una comprensión de los espacios simbólicos que se configuran en la(s) comunidad(es), esto es, una socialidad donde

[...] cada quien, a su manera, compone su ideología, su historia particular, a partir de estos elementos dispares que se encuentran repartidos por todos los rincones del mundo. Estos elementos pueden tomarse prestados de la tradición del lugar o, por el contrario, ser transversales respecto a ésta; sin embargo, sus ensambles presentan similitudes que van a constituir una especie de matriz dando nacimiento y confortando las representaciones particulares. (MAFFESOLI, 2004, p. 140)

En ese espacio social lo importante son las personas, el interactuar dialógicamente en el ethos comunitario, dialogar en sus prácticas culturales, políticas y socioinformativas, revelar la constitución de la identidad de los sujetos y la comunidad, donde la dimensión comunitaria es el momento fundacional de la estructura social. De esta manera la cultura y la política son indisociables

[...] la cultura no puede considerarse como una "instancia exterior" a la política, sino como una dimensión inherente a la vida política o, más precisamente, como una dimensión analítica de todas las prácticas políticas. Esto significa que, lejos de ser 
un decorado accesorio e inesencial, la cultura impregna todo el campo político y "está en todas partes": verbalizada en el discurso, incorporada en las creencias, en los ritos y la teatralización del poder, cristalizada en las instituciones representativas $\mathrm{y}$ en los aparatos de Estado, internalizada en forma de identidades colectivas en conflicto, traducida en forma de ideologías y programas, etc. (GIMÉNEZ, 2007, p. 109)

En este sentido, lo que se persigue es conocer las problemáticas políticas y espacios de poder donde se llevan a cabo prácticas intersubjetivas, valores, actitudes, referencias compartidas, construcciones sociales, constitución de identidades, relaciones de poder en las necesidades de información reales y concretas de los sujetos informacionales en la vida cotidiana del ethos cultural. Como veremos posteriormente, desde esta perspectiva de interpelación de estructuras sociales, se hace una ruptura epistemológica con la noción de usuario de la información, para utilizar ahora la categoría direccionada ontológicamente de sujeto informacional, la cual alude a la pregunta ¿qué es lo que hace ser a este sujeto, un sujeto de la información en un contexto concreto vulnerable que está interpelado por prácticas hegemónicas, ideológicas, discriminatorias, de violencia simbólica, inter alia?

Los sujetos del mundo actual requieren información específica para responder a acontecimientos que los interpelan en la vida cotidiana. Las instituciones sociales del Estado portadoras del saber (escuelas, bibliotecas, museos, etc.) formadoras de sujetos cognoscentes han estado destinadas para el uso exclusivo de un pequeño sector de la población. El acceso democrático al binomio información-conocimiento es una tarea que aún queda pendiente en los gobiernos en turno. En este sentido, es ineludible que se redefina el rol de las unidades de información en su plano institucionalizado -burocrático- para pensar ahora en entidades de información en la comunidad ${ }^{4}$, para que la población -vulnerada y excluida- tenga las condiciones necesarias para ser un sujeto informado y así poder participar en la construcción de una sociedad más justa. Es en este contexto social donde la Bibliotecología, sobre todo la latinoamericana y la mexicana en particular, está en deuda, por lo que le corresponde investigar las problemáticas que demanda la sociedad en un tiempo marcado cada vez más por una creciente desigualdad en toda la estructura de la realidad social y romper con las prácticas ideológicas, y reproductoras del Estado.

En el contexto social contemporáneo, desde un enfoque todavía tradicional, el objetivo disciplinar bibliotecológico ha sido esclarecer los asuntos de acceso a la información

\footnotetext{
${ }^{4}$ Las entidades de información son un espacio democratizador que ofrece espacios de comunicación con otras personas, contemporáneas y del pasado, para construir posibles escenarios. Por tanto no son sólo un fenómeno social y cultural, sino también un importante segmento de la red de comunicación, y su comprensión es esencial para el profesional de la información, cuyo propósito es la de comunicar la información y el saber. (SHERA, 1990)
} 
aprovechando los avances de las TIC. Sin embargo, por la complejidad de la estructura social actual, resulta insoslayable una construcción teórica interdisciplinar de los estudios de la información, principalmente desde lo social, a partir de desarrollar y articular unidades de análisis dentro de diversos marcos teórico-metodológicos, construir herramientas conceptuales para comprender los distintos modos en que el orden social constituye nuestras vidas informativas.

Este tipo de acercamiento a la investigación tiene importantes consecuencias. Ser observador en los diversos contextos comunitarios desde una acción socioprofesional se convierte en una introspección de lo social, lo cual da la posibilidad de construir una perspectiva analítica teórico-metodológica de los problemas de la(s) comunidad(es), productos de las relaciones sociales establecidas por el Estado, y las instituciones.

Es determinante la acción en las comunidades concretas para analizar e identificar las problemáticas y de este modo poder coadyuvar a la transformación del espacio de la dialéctica social: Sujetos (cientista social/sujeto informacional) - Instituciones sociales (entidades de información) - Comunidad.

Para conocer y comprender las necesidades socio-informativas concretas de la(s) comunidad(es) es necesario adentrarse e involucrarse en su contexto, es decir, conocer la comunidad (CONKLIN, 1986) que es la pauta que permite autentificar los fenómenos que acontecen en los espacios sociales. Para ello es necesario estar allí, que según Geertz consiste en dejar constancia de que el investigador estuvo allí, contando de la forma etnográficamente más productiva posible los detalles de su experiencia, identificándose con el entorno social en un proceso empático e inmiscuyéndose en lo que está ocurriendo, lejos del estilo académico, borrando la diferencia entre observador y observado (GEERTZ, 1997). Así pues el estar allí es construir un relato, dar un testimonio sobre los sujetos y la relación con su entorno, al mismo tiempo que proporciona la facultad de dar cuenta de las prácticas sociales, culturales, educativas, informativas, políticas, económicas, religiosas, de usos y costumbres, en suma, de la economía política del intercambio simbólico en el espacio social: capital económico, político, cultural y social (BOURDIEU, 2008).

Un estar allí, consiste tanto en dar un sentido a la realidad de la comunidad, como encontrar el sentido que la comunidad tiene de sí misma y la importancia que adquiere el binomio información-conocimiento en ella, es decir, la construcción de la realidad socioinformativa debe tomar en cuenta las costumbres, los valores, los mitos y tabúes de los grupos comunitarios bajo una visión que incluye la interacción con los grupos sociales, instituciones 
y ethos culturales. Esa relación involucra al sujeto investigador con el objeto de estudio, con la comunidad y la sociedad toda como correspondencia dialógica. Ese tipo de acercamiento a nuestro estudio nos permite realizar como investigadores sociales una intervención en la construcción social de la realidad.

Una de las herramientas que esgrime la EIC son los paradigmas contextuales, que se conciben como una cosmovisión particular de ver la realidad en tiempos y espacios concretos que cuestiona el orden establecido, lo somete a una continua vigilancia crítica y determina alternativas o innovaciones para el ajuste de marcos teóricos, metodologías y uso de herramientas conceptuales. Es decir, los paradigmas contextuales son una perspectiva para ver el espacio social a nivel macro en sus prácticas interpelatorias, lo que posibilita identificar el posicionamiento de los sujetos en la estructura social. Para ello es necesario conocer elementos y momentos de la dialéctica social, es decir, todos aquellos actores sociales que se relacionan e interactúan con el contexto, por ejemplo, el lugar que ocupa el profesional de la información en la estructura social, el lugar que la sociedad le da a la biblioteca, y el lugar que la biblioteca le da a los individuos.

Desde esta posición, es urgente conocer la situación real del lugar donde se centran los flujos informacionales y convergen las necesidades socio-informativas y problemáticas de toda índole de los sujetos en situación de desigualdad, vulnerabilidad y exclusión. Así pues, la EIC examina por medio de los paradigmas contextuales, e. g. el impacto que tiene la sociedad contemporánea en sus distintas manifestaciones: TIC, diversidad cultural, identidad y poder, sobre las dinámicas culturales y sociales de distintos actores de la realidad social: campesinos, indígenas, migrantes, grupos vulnerables; y las consecuencias en la organización, y la gestión de los conocimientos dentro del marco de la dinámica global/local de las entidades de información.

Con este enfoque analítico -teórico-metodológico- de la EIC-I se pretende desarrollar un análisis problematizador con la noción sujeto informacional bajo la premisa ontológica y epistemológica en su contexto sobredeterminado. Así mismo esta perspectiva nos ayuda a aproximarnos a las necesidades informativas concretas como construcción social, es decir, socioinformativas, articulando los discursos, las representaciones sociales, (ARAYA UMAÑA, 2002) los habitus en la economía política del intercambio simbólico (BOURDIEU, 1988; 1984) en el nodo identitario de los sujetos y de las distintas instituciones gubernamentales, empresas, $\mathrm{ONG}^{\prime}$ s, asociaciones civiles, sindicatos, cooperativas, fundaciones, espacios de autogestión, inter alia. 


\section{EL SUJETO INFORMACIONAL}

\subsection{Emergencia del sujeto informacional en la sociedad contemporánea}

Ya hemos mencionado en varias ocasiones el término sujeto informacional, ahora es el momento de des-cubrir su sentido. No es difícil constatar que en el mundo actual la desigualdad tanto entre naciones como habitantes en un mismo país se hace más aguda. Al mismo tiempo se presenta el hecho de que la comunicación en la era de la globalización se da a partir de la implementación de las Tecnologías de Información y Comunicación (TIC), las cuales facilitan los procesos comunicativos y acortan las fronteras, aunque paradójicamente alejan los contactos personales inmediatos. Actualmente, en las llamadas sociedad de la información y del conocimiento, se da una relación impersonal, una era de vacíos dialógicas: el individualismo contemporáneo (LIPOVETSKY, 1988). Esta realidad social sincrónica poco a poco se vuelve a histórica, donde reina el individualismo narcisista, la "cultura de consumo" y declina la "cultura humanística". De ahí la necesidad de manipular conscientemente las tecnologías y no que ellas nos manipulen; de contrarrestar la cultura alienante del siglo XXI.

En este esquema tecno-neoliberal de realidades superficiales y netamente economicistas, el sujeto en calidad de ciudadano de una nación se confronta, se articula social, política, culturalmente con múltiples discursos ${ }^{5}$ de heterogéneas contingencias sociales. Es en esta realidad excluyente, contingente, precaria y contextuada el lugar donde el sujeto realiza sus elecciones para constituirse. Esas elecciones implican construir sitios alternativos al modelo económico imperante; nuevos espacios sociales; otras formas de organización y de acción contextual desde la comunidad para abatir los abismos sociales y de sí mismos; participar en movimientos sociales en contracorriente a este esquema globalizador. Todo ello da como resultado la aparición de nuevos agentes en la forma de ser y hacer, de nuevas identidades sociales híbridas (GARCÍA CANCLINI, 1990). Es decir, que a partir de fuerzas políticas antagónicas se da la posibilidad de constitución del sujeto, que es un punto de ruptura para entrar a los planos de la identificación y de las posiciones en el espacio social. Es en este contexto donde se da la emergencia del sujeto informacional, en un escenario que

\footnotetext{
${ }^{5}$ Desde el Análisis Político de Discurso, el discurso es: “[...] como constelación de significados, como estructura abierta, incompleta y precaria que involucra el carácter relacional y diferencial de los elementos y la posibilidad de construir los significados, que puede ser analizado en el plano paradigmático y en el sintagmático; [...] como significado cuyo soporte material puede ser lingüístico [...] o extralingüístico [...] como construcción social de la realidad [...]". (BUENFIL BURGOS, 2004, p. 262-263)
} 
condiciona, pero simultáneamente donde el sujeto interpela para demandar, construir y articular nuevas estructuras socioinformativas para actuar en esa coyuntura social, como es el caso de las bibliotecas comunitarias por ejemplo, para responder a la necesidades informativas concretas del espacio societal.

Ante este fenómeno social, se reafirma que las identidades no se imponen, se construyen; son producto de las relaciones sociales complejas. De esta manera el individuo no se encuentra supeditado a ninguna identidad específica hegemónica y homogenizadora; las identidades son cambiantes en las coyunturas de lo social histórico, "el gran auge de la identidad se sitúa en la confluencia de varios fenómenos", (DUBET, 1987, p. 519) y los sujetos tienen capacidad relativa de distinción, elección y adscripción identitaria.

El siglo que inicia exige reflexionar para poder fundar una crítica profunda del contexto y de sus instituciones sociales, y encontrarse con la autocrítica, que es la única garantía de los individuos para fundar espacios de transformación social, espacios para la participación popular, para construir discursos críticos, sólidos, políticos y públicos, de hacer el intento de liberarnos (NIETZSCHE, 2000, p. 27). Por lo cual, en este devenir sociohistórico, el sujeto contemporáneo está trazando líneas de proyectos socio-políticos. Todos estos elementos y momentos son puntos donde el espacio simbólico de la información se entreteje como un ámbito de capital social y cultural para la transformación de la realidad inmediata.

Por otro lado, el desorden del mundo no promete un tiempo de bienestar e igualdad social. El capitalismo global contemporáneo avanza sobre terrenos contradictorios, a veces reforzando y coadyuvando el bienestar social, pero otras veces, borrando las identidades, opacando las diferencias culturales, regionales, religiosas, étnicas y de género. Los flujos de información a través de los medio masivos de comunicación producen efectos igualmente indistintos, en ocasiones informando y en otras desinformando.

La disyuntiva del mundo contemporáneo ya no es a favor o en contra de la globalización, sino qué tipo de globalización se busca: una pluricultural, inclusiva y con equidad, o una hegemónica, totalitaria y depredadora. Anclándonos desde la primera posibilidad exige la construcción de una analítica reflexiva y dialógica, de una conciencia crítica de los sujetos capaz de responder a lo complejo del ser social. Ello implica no sólo derecho de acceso al binomio de la información-conocimiento, sino también una apropiación de estos recursos tecnológicos e informáticos que imperan socialmente con gran inequidad. 
Esa construcción de espacios autónomos frente al antagonismo hegemónico permite la emergencia de un sujeto que funda realidades de "identidad cultural autónoma", que es la acción intelectual, política, filosófica, científica y humanística social para la construcción de un pensar crítico en aras de una acción más participativa para la conformación de la ciudadanía, la constitución de sujetos e identidades, de espacios comunitarios sustentables, de autogestión y con autodeterminación.

\subsection{Aproximación conceptual}

A la estructura ontológica del ser humano que hemos denominado "ser informacional", para existir es necesario producir y consumir información. (RENDÓN ROJAS; HERRERA DELGADO, 2010, p. 16) Simultáneamente la producción de la información constituye un hecho sociocultural y político, pues se realiza y siempre se ha realizado en el espacio social. Asimismo la organización de la información es un proceso que se realiza toda la vida. Durante todo el tiempo el sujeto produce información e incrementa su acervo social de conocimiento, es decir, hay una práctica de "apropiación" de la informaciónconocimiento -capital cultural-, por lo cual, el sujeto en el transcurso de la vida cotidiana tiene la necesidad de organizarla para evitar el caos. Sin embargo, esa información y conocimiento es un hecho social dado, es decir, en realidad el sujeto en un primer momento no tiene la autodeterminación de ser libre en su elección cultural, religiosa, informativa, de adscripción identitaria, sino se encuentra condicionado.

Al mismo tiempo, una de las tareas de los profesionales de la información ha sido la de organizar la información documental, pero para ello es primordial conocer a la comunidad de sujetos que demandan información-conocimiento. Es decir, que otra tarea es la investigación de la comunidad, estudiar las realidades informacionales de los espacios concretos en donde los sujetos informacionales de hoy en día requieren y/o están construyendo nuevas formas de organización y estructuras de la información ad hoc a su contexto social, político, educativo, cultural, contracultural, laboral, religioso, ideológico, etcétera.

El planteamiento teórico y conceptual que se ha utilizado para denotar al sujeto que se interrelaciona con los usos de la información, lleva el término, "usuario de la información", que se puede entender como aquel 
una unidad o servicio de información, sino que se abre a todos aquellos que potencialmente pueden valerse de tal unidad o servicio. En este sentido, el uso que se le da a la información corresponde al contexto o comunidad particular en la que está inserto el sujeto o usuario de la información. (HERNÁNDEZ SALAZAR, 2006, p. 213$)^{6}$

La ruptura epistemológica que se hará con esta categoría "usuario de la información" en contraste con el "sujeto informacional" es para ampliar el aspecto sociológico en la constitución del sujeto, como aquel actor social que en el $<<$ uso $>>^{7}$ de la información es interpelado por prácticas hegemónicas, políticas, burocráticas, relaciones de poder, prácticas discriminatorias, entre otras, que afectan sus necesidades socioinformativas por el hecho de encontrarse en espacios complejos, vulnerables, de marginación, de problemas con la ley, que limitan su ser y hacer cotidiano. Ese punto donde subyacen problemáticas de diversos factores socioinformativos no son abordados desde esta categoría de usuarios de la información.

Es así como las cualidades de la información se pueden caracterizar por ser de materialidad simbólico cultural, lo que implica los procesos de producción, transmisión y apropiación de significados en contextos histórico-sociales, comunitarios concretos; y de interacción dialógica entre sujetos, instituciones, comunidad y la sociedad toda.

Para construir la noción de sujeto informacional se alude a la intersección de dos aspectos o elementos de la realidad social: el sujeto y la información; y se analiza la relación dialéctica que cada una de estas dimensiones sujeto-objeto tiene uno con otro. En la realidad esos dos momentos se encuentran entretejidos social y simbólicamente, y aquí sólo se distinguen con fines analíticos. El primer proceso tiene que ver con un segmento que va desde la esfera pensante, emocional, productiva hacia la social y cultural, o sea, con la manera en que el sujeto condiciona a la información en un plano de ethos socio-edu-cultural. El segundo proceso opera en sentido contrario, de la información socio-edu-cultural hacia el sujeto, es decir, la influencia que ejerce el contexto de la información sobre el ámbito de la vida cotidiana del sujeto.

Al emplear esta categoría analítica, se parte de una concepción epistemológica social identitaria con un enfoque dialéctico, es decir, no se trata de estudiar sujetos-objetos aislados y abstractos sino su relación en la concatenación de cambios sustanciales o contingentes de la macro y micro realidad social. Se distingue la incidencia que tiene la actividad socioinformativa en los sujetos, sobre sus ideas, representaciones, valores, normas, rutinas,

\footnotetext{
${ }^{6}$ Véase de la misma autora HERNÁNDEZ SALAZAR, 1983; 2003;2007; 2008

${ }^{7}$ De acuerdo con Wittgenstein, el concepto de $<<$ uso $>>$ hace referencia a la necesidad de contextuar las formas en que se emplean los significantes. El $<<$ uso $>>$ no es lo mismo en distintos enclaves socioculturales, cambia de acuerdo a los contextos de los enunciadores. (WITTGENSTEIN, 1988)
} 
costumbres, tradiciones, planes, fórmulas, reglas, instrucciones, modas, hábitos, lo político, ético en comunidades concretas y sociedad en general.

Se entiende que la parte fundamental de la estructura social son los sujetos, pues son ellos quienes transforman la sociedad, y es a partir de ellos que generan un mundo poblado de satisfacciones y necesidades, entre ellas de información. Es así como los sujetos adquieren el calificativo de "sujeto informacional", el cual se constituye al ser él quien toma en cuenta sus necesidades reales y concretas de información tanto personales como colectivas.

\subsection{Espacio socio-informacional y sujeto informacional}

La noción comunidad, alude a común, por tanto debe haber algo en común entre la interrelación del sujeto y los fenómenos que acontecen socialmente, ya sea para estar de acuerdo o no. Sin embargo, no siempre que existen cosas en común podemos hablar de comunidad, por ejemplo, los asistentes a un espectáculo, comparten un espacio y hasta un interés por lo que están viendo; pero no constituyen comunidad alguna, ni poseen identidades comunes. Por lo anterior es necesario concretar más, lo común debe ser el contexto de interacción -socialidad-, esto es, la identificación del "yo" en el reconocimiento con los “otros" -otredad/alteridad-, a través del mismo idioma, o medio de comunicación, las mismas plataformas, las mismas costumbres, concepciones del mundo, los mismos motivos o un mismo afecto (CASTELLS, 2001). En este sentido, al conceptualizar comunidad es necesario hacer énfasis en su componente cultural -patrones simbólicos- y subrayar la función de apoyo que cumple para los sujetos individuales y sociales los lazos interpersonales que proporcionan sociabilidad, apoyo, un sentimiento de pertenencia y una identidad que interactúa en distintos momentos de la estructura social.

Del planteamiento anterior surgen dos cuestiones, una ¿basta con esto para que se forme una comunidad? y la siguiente cuestión, ¿Se constituyen sujetos informacionales concretos de acuerdo a un contexto? o ¿Se crean escenarios socioinformativos distintos donde convergen distintas identidades personales-sociales?, el cuestionamiento tiene una implicación compleja que sólo puede ser contestada en el estudio concreto de los sujetos y su comunidad.

A continuación se caracteriza grosso modo a los sujetos informacionales y se identifican por el espacio social en el que interactúan junto a otras categorías de sujetos en contextos claves: 


\begin{tabular}{|c|c|c|c|}
\hline \multicolumn{4}{|c|}{ El sujeto informacional y el enclave sociocultural } \\
\hline $\begin{array}{c}\text { Marcos de } \\
\text { identificación }\end{array}$ & $\begin{array}{c}\text { Sujeto informacional. } \\
\text { Por el lugar } \\
\text { geográfico en el que se } \\
\text { encuentra. }\end{array}$ & $\begin{array}{l}\text { Sujeto informacional. } \\
\text { Por conceptos de comunidad. }\end{array}$ & $\begin{array}{c}\text { Sujeto } \\
\text { informacional. } \\
\text { Por status social }\end{array}$ \\
\hline $\begin{array}{l}\text { A. Tipos de } \\
\text { comunidad }\end{array}$ & $\begin{array}{l}\text { Urbana } \\
\text { Suburbana } \\
\text { Rural } \\
\text { Rural-urbana } \\
\text { Industrial } \\
\text { Organización popular } \\
\text { Entre otras } \\
\end{array}$ & & \\
\hline $\begin{array}{l}\text { B. Identificación } \\
\text { categorial }\end{array}$ & & $\begin{array}{l}\text { Religiosa: sacerdote, monjas, } \\
\text { feligreses, catequistas, etc. } \\
\text { Política: Presidente, diputados, } \\
\text { senadores, legisladores, } \\
\text { simpatizantes, etc. } \\
\text { Académica: Investigadores, } \\
\text { docentes, alumnos, etc. } \\
\text { Penitenciaria: Internas e } \\
\text { internos, custodios, profesores, } \\
\text { servidor público, etc. } \\
\text { Popular: Militante, } \\
\text { Colaborador orgánico, } \\
\text { Colaborador eventual. } \\
\text { Etcétera. }\end{array}$ & \\
\hline $\begin{array}{l}\text { C. Estructura de } \\
\text { la comunidad }\end{array}$ & & & $\begin{array}{l}\text { Económica } \\
\text { Cultural } \\
\text { Educativa } \\
\text { Jurídico- } \\
\text { Política } \\
\text { Social } \\
\text { Criminal } \\
\text { Etcétera. }\end{array}$ \\
\hline
\end{tabular}

En este cuadro se expone la categoría sujeto informacional, en un marco de adscripción identitaria que tienen las posiciones de sujeto y para representar el espacio informativo como una estructura institucional, un entorno académico, de organización alternativa y que está abierta a otros posibles enclaves socioculturales emergentes. Es el contexto sujeto e información, donde el significado o representación que puede adquirir la información para los sujetos informacionales en espacios o enclaves particulares es inconmensurable.

El espacio social se articula por el modo en que los sujetos (o grupos) son distribuidos en función de su posición en la estructura social de acuerdo a dos principios de diferenciación: el capital económico y el capital cultural. Los enclaves sociales y culturales representan el conjunto de percepciones, características y prácticas, expresadas individual y/o colectivamente en un territorio o espacio particular de los sujetos en la sociedad. Dichos 
enclaves explican, determinan y condicionan el valor simbólico de un concepto, su significado, en este caso particular, de la representación de las necesidades de información, del acceso a la información y de la representación de las entidades de información (biblioteca) en una comunidad concreta.

La distribución de la economía politica del intercambio simbólico en el espacio socioinformacional está dada por las posiciones (campo) y por la disposición (lógica de interacción) de los sujetos en las interacciones sociales de la estructura, es decir, por la predeterminación de lo social en los sujetos, "por un campo de fuerza que impone su determinación específica en todos aquellos que entran en él”. (WACQUANT, 2005, p. 64)

El habitus es la suma de valores, las formas de ser socialmente, un repertorio de condiciones posibles para la acción. La posición de cualquier individuo, grupo o institución en el espacio social puede entonces ubicarse mediante dos coordenadas: el volumen total y la composición del capital que detentan. Una tercera coordenada, la variación en el tiempo de este volumen y de esta composición, registra su trayectoria a través del espacio social y brinda claves invaluables acerca de su habitus al revelar la manera y la senda por medio de las cuales alcanzaron la posición que ocupan en el presente. (WACQUANT, 2005, p. 63)

Así pues al desarrollar investigaciones en Bibliotecología es necesario conocer el espacio social de los sujetos informacionales de acuerdo al paradigma contextual; sus niveles de apropiación de capitales económico y cultural; sus enclaves sociales y culturales, sus posiciones y disposiciones en las interacciones sociales y sus habitus.

\section{REFLEXIONES FINALES}

El sujeto informacional se constituye al tener una posición en el espacio socioinformacional concreto, al observar, reflexionar, analizar y actuar en la estructura social bajo una crítica profunda, al ser poseedor de un capital de información de coyuntura social, como un acto político para denunciar que el status quo contemporáneo requiere de una restructuración; a partir del sentimiento de comunidad y reconocimiento del otro.

Existir en un mundo poblado de desigualdad e injusticia hace patente la existencia de principios ideológicos hegemónicos que practican políticas excluyentes. Al anclarse el sujeto informacional con el contexto surge un efecto de interpelación que es la vía para la constitución identitaria. 
La constitución del sujeto informacional desde el espacio comunitario involucra una manera de acción para resolver problemas, de dar una respuesta colectiva a problemas colectivos, una tarea transformadora de la realidad social, es un reconocimiento para armar un conjunto de estrategias informativas ad hoc al contexto social que interpela. Esta acción comunitaria es necesaria en lo político, lo social, lo económico, lo educativo, en general, lo cultural para generar comunidad, si es preciso, comunidad-red mediante la apropiación de las TIC como recursos dialogantes, su empleo en entidades de información.

Las acciones comunitarias bajo el sujeto informacional implican la generación de mecanismos informativos para la autogestión, la autoformación y emancipación.

La configuración analítica de la Epistemología de la Identidad Comunitaria Informacional (EICI), propone la manera de pensar y de abordar la realidad informativa desde una perspectiva de praxis y dialéctica social (ontológica, epistemológica y metodológicamente,) por medio de sus categorías ex profeso, i.e. paradigma contextual, sujeto informacional que abren amplias y fecundas posibilidades para estudiar distintos fenómenos que ocurren en las entidades de información (contingencias, rupturas, burocratismo, rezagos, discriminación, exclusión, violencia, entre otras patologías sociales) de la realidad socio-informativa en contextos vulnerables.

De los argumentos anteriores se desprende que la constitución del sujeto informacional está interpelado por las prácticas de un sistema hegemónico y está sobredeterminado por una multiplicidad de articulaciones informacionales -políticas de acceso a la información-, pero que a su vez este sujeto interpela y da posibilidad a su identidad por medio del antagonismo a un contexto social concreto. Escenario social donde subyacen diversos matices socioinformativos, por ende, necesidades informativas definidas de acuerdo al interregno político-cultural.

\section{REFERÊNCIAS}

ARAYA UMAÑA, Sandra. Las representaciones sociales: ejes teóricos para su discusión. Costa Rica: Facultad Latinoamericana de Ciencias Sociales, 2002.

BERGER, Peter L., LUCKMANN, Thomas. Modernidad, pluralismo y crisis de sentido: la orientación del hombre moderno. España: Paidós, 1997.

BOURDIEU, Pierre. Capital cultural, escuela y espacio social. $8^{\mathrm{a}}$ ed. México: Siglo XXI, 2008. 
. La distinción. Criterios y bases sociales del gusto. Madrid: Taurus, 1988.

. Sociología y cultura. Barcelona: Gedisa, 1984.

BUENFIL BURGOS, Rosa Nidia. Argumentación y poder: La mística de la Revolución Mexicana. México: Plaza y Valdés, 2004.

BUENFIL BURGOS, Rosa Nidia. La categoría intermedia. In: Investigación social. Herramientas teóricas y Análisis Político de Discurso. México, Casa Juan Pablos, Programa de Análisis de Discurso e Investigación, 2008.

BUSHA, Charles H.; HARTER, Stephen P. (1990) Métodos de investigación en Bibliotecología: técnicas e interpretación. México: UNAM, 1990.

CASTELLS, Manuel. La galaxia Internet. Barcelona: Arete, 2001.

CONKLIN, Harold C. Etnografía. Ediciones Pirata, México: Universidad de Veracruz. Núm. 96, 1986.

DUBET, François. De la sociología de la identidad a la sociología del sujeto. Estudios Sociológicos, México: Colegio de México. 7 (21) sept- dic, 1987.

GARCÍA CANCLINI, Nestor. Culturas híbridas: estrategias para entrar y salir de la modernidad. México: Grijalbo, CONACULTA. 1990. 363 p.

GARCÍA CERVANTES, Luis Alejandro. Epistemología de la Identidad Comunitaria Informacional. Análisis teórico metodológico para el estudio de la dialéctica social bibliotecológica. Ciudad de México: Universidad Nacional Autónoma de México, 2010. (Tesis de Maestría en el Posgrado de Bibliotecología y Estudios de la Información).

GEERTZ, Clifford. Estar allí. La antropología y la escena de la escritura. In: EI antropólogo como autor. España: Paidós, 1997.

GIMÉNEZ, Gilberto. Cultura política e identidad. In: Estudios sobre la cultura y las identidades sociales. México. CONACULTA, ITESO, 2007.

HERNÁNDEZ SALAZAR, Patricia. Análisis metodológico para abordar el fenómeno usuarios de la información en América Latina. Investigación Bibliotecológica. México: UNAM, CUIB. v. 17, n. 35 jul-dic, p. 5-31, 2003.

El perfil del usuario de información. Investigación Bibliotecológica: archivonomía, bibliotecología e información. México: UNAM, CUIB. v. 7. n. 5. pp. 16-22, 1983.

El usuario de la información. In: Servicios bibliotecarios / coord. Hugo Alberto Figueroa Alcántara y César Augusto Ramírez Velázquez. México: UNAM, Facultad de Filosofía y Letras; Dirección General de Asuntos del Personal Académico. 2006, p. 209-227.

. La relación entre los estudios y la formación de usuarios de la información. Revista General de Información y Documentación. v. 17. n. 2. pp. 103-121, 2007. 
et al. Métodos cualitativos para estudiar a los usuarios de la información.

Patricia Hernández Salazar, et. al. México: UNAM, CUIB, 2008.

LIPOVETSKY, Guilles. La era del vacío: ensayos sobre el individualismo contemporáneo. Barcelona: Anagrama, 1988.

LYOTARD, Jean François. La condición postmoderna: informe sobre el saber. México: Rei, 1990.

LYOTARD, Jean François. La postmodernidad: explicada a los niños. Barcelona: Gedisa, 1987.

MAFFESOLI, Michel. EI tiempo de las tribus: el ocaso del individualismo en las sociedades posmodernas. México: Siglo XXI, 2004.

NIETZSCHE, Friedrich. Shopenhauer como educador. España: Biblioteca nueva, 2000.

RENDÓN ROJAS, Miguel Ángel; HERRERA DELGADO, Lizbeth Berenice. Bases filosóficas de la organización de la información. Perspectivas em ciência da informação. $v$. 15, n. 1. Belo Horizonte, p. 3-17, 2010. Disponível em:

$<$ http://www.scielo.br/scielo.php?script=sci_arttext\&pid=S1413-

99362010000100002\&lng=en\&nrm=iso $>$ Acessado em: 01 set. 2011.

SHERA, Jesse. H. Los fundamentos de la educación bibliotecológica. México: UNAM, CUIB, 1990.

TOURAINE, Alain. Crítica de la modernidad. 2a ed. México: FCE, 2000.

VATTIMO, Gianni. et al. En torno a la posmodernidad.Barcelona: Anthropos, 1990.

WACQUANT, Loïc. Claves para leer a Bourdieu. In: Ensayos sobre Pierre Bourdieu y su obra. Isabel Jiménez (Coord.). México, UNAM, CESU, Plaza y Valdés. 2005, p. 53-78

WITTGENSTEIN, Ludwig. Investigaciones filosóficas. UNAM, Instituto de Investigaciones Filosóficas; Barcelona: Crítica, 1988. 